PROCEEDINGS OF THE

AMERICAN MATHEMATICAL SOCIETY

Volume 128, Number 3, Pages 671-675

S 0002-9939(99)05206-5

Article electronically published on July 6, 1999

\title{
MATRICES OVER ORDERS IN ALGEBRAIC NUMBER FIELDS AS SUMS OF $k$-TH POWERS
}

\author{
S. A. KATRE AND SANGITA A. KHULE \\ (Communicated by David E. Rohrlich) \\ Dedicated to the memory of David R. Richman
}

\begin{abstract}
David R. Richman proved that for $n \geq k \geq 2$ every integral $n \times n$ matrix is a sum of seven $k$-th powers. In this paper, in light of a question proposed earlier by M. Newman for the ring of integers of an algebraic number field, we obtain a discriminant criterion for every $n \times n$ matrix $(n \geq k \geq 2)$ over an order of an algebraic number field to be a sum of (seven) $k$-th powers.
\end{abstract}

\section{INTRODUCTION}

M. Newman [1] showed that, for $n \geq 2$, every matrix in $M_{n} \mathbf{Z}$ is a sum of 7 or 9 squares according as $n$ is even or odd. He then posed the problem for the ring of integers (i.e. the maximal order) of an algebraic number field.

Vaserstein [3], [4] showed that every integral $n \times n$ matrix $(n \geq 2)$ is a sum of three squares by proving the following

Theorem A. A matrix $A$ in $M_{n} R$ ( $R$ a commutative ring with 1 and $n \geq 2$ ) is a sum of squares if and only if $A$ is a sum of three squares if and only if tr. $A \equiv$ square $(\bmod 2 R)$.

David R. Richman [2] showed that, for $n \geq k \geq 2$, every $n \times n$ integral matrix is a sum of seven $k$-th powers using his following key-result:

Theorem B. Let $n \geq 2, R$ a commutative ring with 1 . The following are equivalent:

(i) $M$ is a sum of $k$-th powers in $M_{n} R$.

(ii) $M$ is a sum of seven $k$-th powers in $M_{n} R$.

(iii) $M \in M_{n} R$ and for every prime power $p^{e}$ dividing $k$, there are elements $x_{0}=x_{0}(p), \cdots, x_{e}=x_{e}(p)$ in $R$, such that

$$
\text { tr.M }=x_{0}^{p^{e}}+p x_{1}^{p^{e-1}}+p^{2} x_{2}^{p^{e-2}}+\cdots+p^{e} x_{e} .
$$

If $F$ is a field of characteristic 0 , then it follows from Theorems $\mathrm{A}$ and $\mathrm{B}$ that every matrix in $M_{n} F(n \geq 2)$ is a sum of three squares, and for $n \geq k \geq 2$, every matrix in $M_{n} F$ is a sum of seven $k$-th powers in $M_{n} F$. The same result also follows

Received by the editors April 21, 1998.

1991 Mathematics Subject Classification. Primary 11P05, 11R04, 15A33; Secondary 11C20, 11E25, 15A24.

Key words and phrases. Algebraic number fields, order, sums of powers, discriminant, matrices.

(C)1999 American Mathematical Society 
for matrices over $\mathbf{Z}$. However, if we consider the ring of integers or other orders in algebraic number fields, then we find that for some of these rings such a result is true, whereas for some other rings we get counter-examples. For instance, if $R=\mathbf{Z}[i]$, then $R / 2 R=\{\overline{0}, \overline{1}, \bar{i}, \overline{1+i}\}$. Here $\overline{0}$ and $\overline{1}$ are the only squares, and an $n \times n$ matrix over $\mathbf{Z}[i](n \geq 2)$ whose trace is $\equiv i$ or $1+i(\bmod 2 R)$ is not a sum of squares in $\mathbf{Z}[i]$. On the contrary, every element of $R / 3 R$ is a cube, so every $n \times n$ matrix $(n \geq 3)$ over $\mathbf{Z}[i]$ is a sum of seven cubes. One has exactly the reverse situation for $R=\mathbf{Z}[\omega], \omega=\exp (2 \pi i / 3)$, and we get that every $n \times n$ matrix $(n \geq 2)$ over $\mathbf{Z}[\omega]$ is a sum of 3 squares, but for every $n \geq 3$, we find matrices over $\mathbf{Z}[\omega]$ which are not sums of cubes in $\mathbf{Z}[\omega]$.

In this paper, we take up this problem (earlier raised by Newman) for orders in algebraic numbers fields and obtain the following discriminant criterion:

Theorem 1. Let $R$ be an order in an algebraic number field $K$. Let $n \geq k \geq 2$. Then every $n \times n$ matrix over $R$ is a sum of (seven) $k$-th powers if and only if $(k$, disc. $R)=1$.

\section{Matrices OVER the Ring of integers OF AN ALGEBRAIC NUMBER FIELD}

Henceforth, let $K$ denote an algebraic number field and $\mathbf{O}$ the ring of integers of $K$. The discriminant of $K$ or the discriminant of $\mathbf{O}$ denotes the discriminant of any integral basis of $K$ (i.e. a $\mathbf{Z}$-basis of $\mathbf{O}$ ). In this section we prove

Proposition 1. Let $n \geq k \geq 2$. Every $n \times n$ matrix over $\mathbf{O}$ is a sum of (seven) $k$-th powers if and only if $(k$, disc.K $)=1$.

For this, we first note the following lemmas:

Lemma 1. Let $R$ be a commutative ring with 1 . Let $p$ be a prime. The following are equivalent:

(i) Every element of $R$ is a $p$-th power $(\bmod p R)$.

(ii) For every $e \geq 1$, given any $x \in R$, there are elements $x_{0}, x_{1}, \cdots, x_{e}$ depending upon $p$ and e such that

$$
x=x_{0}^{p^{e}}+p x_{1}^{p^{e-1}}+p^{2} x_{2}^{p^{e-2}}+\cdots+p^{e} x_{e} .
$$

Proof. (ii) $\Rightarrow$ (i) is clear. To prove (i) $\Rightarrow$ (ii), first prove by induction that $a \equiv$ $b(\bmod p R) \Rightarrow a^{p^{i}} \equiv b^{p^{i}}\left(\bmod p^{i+1} R\right)$. Then (ii) can be proved by induction by noting that if $x_{i} \equiv y_{i}^{p}(\bmod p R)$, then $x_{i}^{p^{e-i}} \equiv y_{i}^{p^{e-i+1}}\left(\bmod p^{e-i+1} R\right)$ so that $p^{i} x_{i}^{p^{e-i}} \equiv$ $p^{i} y_{i}^{(e+1)-i}\left(\bmod p^{e+1} R\right)$.

Lemma 2. Let $R$ be a commutative ring with unity. Let $n \geq k \geq 2$. The following are equivalent:

(1) Every matrix in $M_{n} R$ is a sum of $k$-th powers in $M_{n} R$.

(2) Every matrix in $M_{n} R$ is a sum of seven $k$-th powers in $M_{n} R$.

(3) For every $p$ dividing $k$, every element of $R$ is a $p$-th power $(\bmod p R)$.

Proof. (1) $\Leftrightarrow(2)$ is due to Theorem B of Richman. (1) $\Leftrightarrow$ (3) is obtained by combining Theorem $\mathrm{B}$ and Lemma 1 , and by noting that every $x \in R$ is the trace of the diagonal matrix diag. $\{x, 0,0, \cdots, 0\}$. 
Lemma 3. Let $p$ be a prime. The following are equivalent:

(i) Every element of $\boldsymbol{O}$ is a $p$-th power $(\bmod p \mathbf{O})$.

(ii) $(p$, disc. $K)=1$.

Proof. $\quad(\Leftarrow)$ Let $(p$,disc. $K)=1$. Then $p$ is unramified in $K$, so $p \mathbf{O}=\wp_{1} \wp_{2} \cdots \wp_{r}$, where $\wp_{1}, \wp_{2}, \cdots, \wp_{r}$ are distinct primes of $\mathbf{O}$. Then by the Chinese remainder theorem, $\mathbf{O} / p \mathbf{O} \approx \mathbf{O} / \wp_{1} \oplus \cdots \oplus \mathbf{O} / \wp_{r}$. Each $\mathbf{O} / \wp_{i}$ is a finite field of characteristic $p$, so every element of $\mathbf{O} / \wp_{i}$ and hence of $\mathbf{O} / p \mathbf{O}$ is a $p$-th power.

$\Leftrightarrow)$ Suppose $p \mid$ disc.K. Then $p$ is ramified in $K$. Let $\wp$ be a prime divisor of $p$ which ramifies. Take $x \in \wp$ such that $x \notin \wp^{2}$. Then $x \not \equiv y^{p}(\bmod p \mathbf{O})$ for any $y \in \mathbf{O}$. For otherwise, $\wp|x \Rightarrow \wp| y^{p} \Rightarrow \wp\left|y \Rightarrow \wp^{2}\right| x\left(\right.$ as $\left.\wp^{2} \mid p \mathbf{O}\right)$, a contradiction.

Proof of Proposition 1. Follows by combining Lemma 2 and Lemma 3.

Corollary 1. Let $m$ be a squarefree integer. Let $\mathbf{O}$ be the ring of integers of $K=$ $\mathbf{Q}(\sqrt{ } m)$ (i.e. $\mathbf{O}=\mathbf{Z}[\sqrt{ } m]$ if $m \equiv 2,3(\bmod 4)$ and $\mathbf{O}=\mathbf{Z}[(1+\sqrt{ } m) / 2]$ if $m \equiv 1$ $(\bmod 4)$.) Let $n \geq k \geq 2$. Then every matrix in $M_{n} \mathbf{O}$ is a sum of (seven) $k$-th powers if and only if $(k, m)=1$ and either

(i) $k$ is odd, or

(ii) $k$ is even and $m \equiv 1(\bmod 4)$.

Proof. Disc. $\mathbf{Q}(\sqrt{ } m)= \begin{cases}m, & \text { if } m \equiv 1(\bmod 4), \\ 4 m, & \text { if } m \equiv 2,3(\bmod 4) .\end{cases}$

Corollary 2. Let $m \geq 1$ and $\zeta_{m}$ be a primitive $m$-th root of unity. The ring of integers of the cyclotomic field $K=\mathbf{Q}\left(\zeta_{m}\right)$ is $\mathbf{O}=\mathbf{Z}\left[\zeta_{m}\right]$.

Let $n \geq k \geq 2$. Then every $n \times n$ matrix over $\mathbf{Z}\left[\zeta_{m}\right]$ is a sum of (seven) $k$-th powers if and only if either

(i) $(k, m)=1$, or

(ii) $m \equiv 2(\bmod 4)$ and $(k, m)=2$.

Proof. Note that $\mathbf{Q}\left(\zeta_{m}\right)=\mathbf{Q}\left(\zeta_{2 m}\right)$, if $m$ is odd. Also for $m \not \equiv 2(\bmod 4)$, the prime divisors of disc. $\mathbf{Q}\left(\zeta_{m}\right)$ are the same as the prime divisors of $m$.

\section{MATRICES OVER ORDERS IN ALGEBRAIC NUMBER FIELDS}

An order in a algebraic number field $K$ is a ring containing 1 , and which is a finitely generated $\mathbf{Z}$-submodule of $K$ of maximum rank, i.e. of $\operatorname{rank} N=\operatorname{deg}(K / \mathbf{Q})$. One notes that $\mathbf{O}$ is an order of $K$ and $\mathbf{O}$ contains every order; hence $\mathbf{O}$ is called the maximal order of $K$. The discriminant of an order $R$ is defined to be the discriminant of any $\mathbf{Z}$-basis of $R$.

Lemma 4. If $K$ is a number field of degree $N$, $\mathbf{O}$ the ring of integers of $K$, and $R$ an order of $K$, then there are $a \mathbf{Z}$-basis $\theta_{1}, \theta_{2}, \cdots, \theta_{N}$ of $\mathbf{O}$ and $a \mathbf{Z}$-basis $\alpha_{1}, \alpha_{2}, \cdots, \alpha_{N}$ of $R$ such that $\alpha_{i}=f_{i} \theta_{i}, f_{i} \in \mathbf{Z}, f_{i}>0$, and moreover

$$
f_{1}\left|f_{2}\right| \cdots \mid f_{N} \text {. }
$$

Proof. Start with any $\mathbf{Z}$-bases $\eta_{1}, \cdots, \eta_{N}$ and $\beta_{1}, \cdots, \beta_{N}$ of $\mathbf{O}$ and $R$ respectively. Let $A \in M_{N} \mathbf{Z}$ such that $\left[\beta_{1}, \cdots, \beta_{N}\right]=\left[\eta_{1}, \cdots, \eta_{N}\right] A$. As $\mathbf{Z}$ is a PID, there exist unimodular matrices $P$ and $Q$ such that $P A Q=$ diag. $\left[f_{1}, f_{2}, \cdots, f_{N}\right]$ is the Smith normal form of $A$, so that $f_{1}\left|f_{2}\right| \cdots \mid f_{N}$. As rank of $A$ is $N$, each $f_{i} \neq 0$. We 
may also assume that each $f_{i}>0$. Now

$$
\left[\beta_{1}, \cdots, \beta_{N}\right] Q=\left[\eta_{1}, \cdots, \eta_{N}\right] P^{-1}(P A Q) .
$$

Call $\left[\beta_{1}, \cdots, \beta_{N}\right] Q=\left[\alpha_{1}, \cdots, \alpha_{N}\right]$ and $\left[\eta_{1}, \cdots, \eta_{N}\right] P^{-1}=\left[\theta_{1}, \cdots, \theta_{N}\right]$.

Lemma 5. With the bases of $\mathbf{O}$ and $R$ as in Lemma 4, one has

$$
\text { index of } R \text { in } \mathbf{O}=f_{1} f_{2} \cdots f_{N} \text { and disc. } R=\left(f_{1} f_{2} \cdots f_{N}\right)^{2} \text { disc. } K .
$$

Proof. Clear.

Lemma 6. Let $R$ be a commutative ring with 1 . Let $p$ be a prime and $R / p R$ a finite ring. The following are equivalent:

(i) Every element of $R$ is a $p$-th power $(\bmod p R)$.

(ii) $x \in R, x^{p} \in p R \Rightarrow x \in p R$.

Proof. Let the map $\phi: R / p R \rightarrow R / p R$ be given by $\alpha \rightarrow \alpha^{p}$. Then $\phi$ is a homomorphism. Now

(i) $\Leftrightarrow \phi$ is onto $\Leftrightarrow \phi$ is one-one (as $R / p R$ is finite) $\Leftrightarrow \operatorname{ker} \phi$ is trivial $\Leftrightarrow$ (ii).

Proof of Theorem 1. $(\Leftarrow)$ Suppose $(k$, disc.R $)=1$. Let $p$ be any prime divisor of $k$. Then $(p$, disc. $R)=1$, and by Lemma $5,(p$, disc. $K)=1$. As $R / p R$ is finite, in view of Lemmas 2 and 6 , it suffices to prove that $x \in R, x^{p} \in p R \Rightarrow x \in p R$. Thus let $x \in R, x^{p} \in p R$. Then $x^{p} \in p \mathbf{O}$. As $(p$, disc. $K)=1$, by Lemma 3 and Lemma 6 , $x \in p \mathbf{O}$. Let $\theta_{1}, \cdots, \theta_{N}$ and $\alpha_{1}, \cdots, \alpha_{N}$ be bases of $\mathbf{O}$ and $R$ respectively, chosen as in Lemma 4. As $x \in R$, let $x=\sum_{i=1}^{N} a_{i} \alpha_{i}$. Then $x=\sum_{i=1}^{N} a_{i} f_{i} \theta_{i}$. As $x \in p \mathbf{O}$, there is $b_{i} \in \mathbf{Z}$ such that $a_{i} f_{i}=p b_{i}(1 \leq i \leq N)$. As $(p$, disc. $R)=1$, by Lemma 5 , $\left(p, f_{i}\right)=1$, so $p \mid a_{i}$ for each $i$. Hence $x \in p R$.

$(\Rightarrow)$ Suppose $(k$, disc. $R) \neq 1$. Let $p$ be a prime such that $p \mid(k$, disc.R $)$. Now, disc. $R=\left(f_{1} \cdots f_{N}\right)^{2}$ disc.K.

Case (i). Suppose $\left(p, f_{i}\right)=1$ for all $1 \leq i \leq N$. Then $p \mid$ disc.K.

Assume, for contradiction, that every matrix in $M_{n} R$ is a sum of $k$-th powers. Then by Lemma 2, every element of $R$ is a $p$-th power $\bmod p R$, say $\alpha_{i} \equiv \gamma_{i}^{p}$ $(\bmod p R)$. Let $b_{i}, c_{i} \in \mathbf{Z}$ such that $1=b_{i} p+c_{i} f_{i}$. Then $\theta_{i}=b_{i} p \theta_{i}+c_{i} f_{i} \theta_{i} \equiv$ $c_{i} \alpha_{i} \equiv c_{i}^{p} \gamma_{i}^{p}(\bmod p \mathbf{O})\left(\right.$ noting that $\left.c_{i} \equiv c_{i}^{p}(\bmod p \mathbf{Z})\right)$. Thus if $x=\sum_{i=1}^{N} a_{i} \theta_{i} \in$ $\mathbf{O}$ with $a_{i} \in \mathbf{Z}$, then $x \equiv\left(\sum a_{i} c_{i} \gamma_{i}\right)^{p}(\bmod p \mathbf{O})$. This gives $(p$, disc.K) $=1$, by Lemma 3. Contradiction.

Case (ii). Suppose $p \mid f_{j}$ for some $j$. Due to the choice of the $f_{i}$ 's as in Lemma 4, we have $f_{1}\left|f_{2}\right| \cdots \mid f_{N}$, so $p \mid f_{N}$. Also, $f_{N} \theta_{i} \in R$ for all $1 \leq i \leq N$, and so $f_{N} \alpha \in R$, for every $\alpha \in \mathbf{O}$. In particular $\beta=f_{N}\left(f_{N}^{p-2} \theta_{N}^{p}\right) \in R$. As $p \mid f_{N}, \alpha_{N}^{p}=f_{N} \beta \in p R$. However, $\alpha_{N} \in p R$, as $\alpha_{1}, \cdots, \alpha_{N}$ is a $\mathbf{Z}$-basis of $R$. Hence from Lemma 2 it follows that there are matrices in $M_{n} R$ that are not sums of $k$-th powers in $M_{n} R$.

Remark 1. Let $(k$, disc. $R)>1$. Let $p$ be the smallest prime divisor of $(k$, disc.R). Then, for every $n \geq p$, there are $n \times n$ matrices (which are not sums of $p$-th powers, and hence) which are not sums of $k$-th powers in $M_{n} R$.

Remark 2. Combining Theorem A and Theorem 1, we see that for an order $R$, if disc. $R$ is odd (i.e. $\equiv 1(\bmod 4))$, then for every $n \geq 2$, every matrix in $M_{n} R$ is a sum of three squares. Also, if disc. $R$ is even (i.e. $\equiv 0(\bmod 4))$, then for every $n \geq 2$, there are matrices in $M_{n} R$ which are not sums of squares in $M_{n} R$. 
Corollary 3. Let $m$ be a squarefree integer, and $f$ denote a positive integer. If $m \equiv 1(\bmod 4)$, the orders of $\mathbf{Q}(\sqrt{ } m)$ are $R_{f}=\mathbf{Z}+f((1+\sqrt{ } m) / 2) \mathbf{Z}$. If $m \equiv$ $2,3(\bmod 4)$, the orders are $R_{f}=\mathbf{Z}+f \sqrt{ } m \mathbf{Z}$. Then for $n \geq k \geq 2$ every $n \times n$ matrix over $M_{n} R_{f}$ is a sum of (seven) $k$-th powers if and only if $(k, f m)=1$ and either (i) $k$ is odd or (ii) $k$ is even and $m \equiv 1(\bmod 4)$.

Proof. If $m \equiv 1(\bmod 4)$, disc. $R_{f}=f^{2} m$.

If $m \equiv 2,3(\bmod 4)$, disc. $R_{f}=4 f^{2} m$.

Example 1. Disc. $\mathbf{Z}[\sqrt{ } m]=4 m$, when $m$ is not a perfect square. Hence for every $n \geq 2$, there are matrices over $\mathbf{Z}[\sqrt{ } m]$ which are not sums of squares in $M_{n} \mathbf{Z}[\sqrt{ } m]$ (although they have to be sums of three squares in $M_{n} \mathbf{Q}(\sqrt{ } m)$ ).

Example 2. Disc.Z $\mathbf{i}]=-4$, so for every $n \geq 2$, there are $n \times n$ matrices over $\mathbf{Z}[i]$, which are not sums of squares. Hence for $k$ even, for every $n \geq 2$, there are $n \times n$ matrices over $\mathbf{Z}[i]$ which are not sums of $k$-th powers. However, for $k$ odd, for every $n \geq k$, every $n \times n$ matrix over $\mathbf{Z}[i]$ is a sum of seven $k$-th powers.

\section{REFERENCES}

[1] M. Newman, Sums of squares of matrices, Pacific J. Math. 118 (1985), 497-506. MR 86k:15011

[2] D. R. Richman, The Waring problem for matrices, Linear and Multi. Alg. 22(1987), 171-192. MR 89d:11087

[3] L. N. Vaserstein, Every integral matrix is a sum of three squares, Linear and Multi. Alg. 20(1986), 1-4. MR 88e:15009

[4] L. N. Vaserstein, On the sum of powers of matrices, Linear and Multi. Alg. 21 (1987), 261-270. MR 89a: 15016

Department of Mathematics, University of Pune, Pune-411007, India

E-mail address: sakatre@math.unipune.ernet.in 